\title{
Hepatosplenic T-Cell Lymphoma: Case Report \& Literature Review
}

\author{
Maryam Radi Alghaza* and Abdullah Al Rashed \\ Hematology Consultant, Department of \\ Hematopathology, Regional Laboratory in Dammam \\ (DRL), Saudi Arabia \\ *Correspondling author: Maryam Radi Alghaza, \\ Department of Hematopathology, Regional Laboratory in \\ Dammam (DRL), Saudi Arabia
}

Received: August 01, 2017; Accepted: September 05, 2017; Published: September 26, 2017

\begin{abstract}
Acute myeloid leukemia with Megakaryoblastic and Basophilic differentiation and CML with concurrent Megakaryoblastic and Basophilic Blast crisis are very rare diseases with only few reported cases in the literature.

Diagnosis of this leukemia with two types of blasts of the same lineage can be very challenging and morphology alone is not sufficient especially when the morphology is not classical. Flowcytometry and cytogenetic studies are important to establish the diagnosis. Here we report a case of AML with megakaryoblastic \& basophilic differentiation \& Positive Philadelphia chromosome by FISH.
\end{abstract}

Keywords: Hepatosplenic T-Cell lymphoma; Acute myeloid leukemia; Basophilia; Chronic myelogenous leukemia

\section{Introduction}

Basophilia is commonly associated with Chronic Myelogenous Leukemia, notably in the accelerated phase or during blast crisis. It is also associated with other Myelo proliferative neoplasms. However, its association with acute leukemia is very rare and is described in association with either Acute basophilic leukemia or in Acute Leukemias with a mixture of Blasts with an important participation of mature or Immature Basophilic cells, Like Acute Myeloid Leukemias (AMLs) with recurrent genetic abnormalities such as $\mathrm{t}(6 ; 9)(\mathrm{p} 23 ; \mathrm{q} 34)$. Incidence of $\mathrm{Ph}$ positive AML appears to be very low, apparently constituting less than $1 \%$ of all newly diagnosed cases of AML $[1,2]$ Generally, presence of $\mathrm{Ph}$ chromosome provides strong evidence for blast crisis arising in the background of CML [3]. The most recent published case of similar morphological combination but negative for ph chromosome was published by Sreedharanunni et al in 2016 [4].

\section{Case Presentation}

A 54-year-old Egyptian man, who presented with Anemia and lytic lesion as evident by $\mathrm{x}$-ray, was referred to our institution for Flowcytometry.

Periperhal Blood shows WBC $1717 \times / \mathrm{ul}$. Hb- 6.7g/dl and Platelets count $82,000 /$ ul. Pancytopenia with infiltration by around $14 \%$ Blasts, which are large in size with high Nucleo cytoplasmic ratio, Nucleus is predominantly round to oval with 1-2 nucleoli and mild to moderate amount of basophilic cytoplasm. Many of them have vacuoles. About 2-4 Blasts were slightly smaller in size than others had dark basophilic cytoplasm and had cytoplasmic Blebs (Figure 1).

Neutrophils showed left shift with presence of Metamyelocyte and Myelocyte constituting about $9 \%$. Basophils about $2 \%$ seen. Some Large and Giant Platelets almost twice the size of RBC are also seen. Bone marrow examination revealed. A particulate Hemodiluted Bone Marrow with about $42 \%$ Blasts. The Blasts Morphology is similar to that described in peripheral Smear. About 8 Blasts out of the total Blasts showed slightly smaller size and presence of cytoplasmic Blebs. Myelopoeisis is sequential and constitutes approximately
40\%. Megakaryocytes are present and some of them are Small and Monolobated. Occasional Erythroid element seen.

\section{Sudan black stain}

Negative in Blasts: Flowcytometry done from Bone Marrow Sample sent reveals two Blast Populations. Blast Population- I constitutes about $10 \%$ and has moderate CD45 (Less Brighter than Lymphocytes and Brighter than other Blast population-Basophilic gate) and is positive for Following Markers-CD34, CD117, HLADR, CD13, CD33, CD9, CD22, CD25, and Partial Positive for CD38, CD11c, CD7, CD71-It is consistent with Basophil Blasts. Blast Population-II constitutes about $24 \%$ and has moderate CD 45 , Low SSC and expresses the Following Markers - CD34, CD117, HLA-DR, CD13, CD9, CD71, CD36, and Partial Positive for CD22, s-CD41a-17\%, s-CD61-30\%, and c-CD61-15\%. Consistent with Megakaryoblast phenotype (Figure 2).

Negative marker for both population: CD10, CD19, CD20, c-CD3, c- CD79a, CD11b, CD16, CD64, CD14, CD56, Gly-A, TdT, c-MPO.

Cytogenetic studies: with the use of dual colour dual fusion probe showed $t(9 ; 22)$ ( $q 34 ;$ q11.2), in $24.3 \%$ (68 out of 288 interphase nuclei examined).

\section{Discussion}

In this case morphological findings on peripheral blood and bone marrow are challenging and the distinction between two populations of Blasts was made by Immuno phenotype. There was no prior history of any Myelo proliferative neoplasm nor any presence of Splenomegaly.

However the Divergent differentiation of blasts along with positivity of FISH for Philadelphia chromosome made the diagnosis difficult to determine whether its. De novo Acute Myeloid Leukemia with Philadelphia chromosome positive or it is CML in Blast crisis with concurrent Megakayoblastic and Basophilic differentiation.

The 2016 revision of the World Health Organization classification 


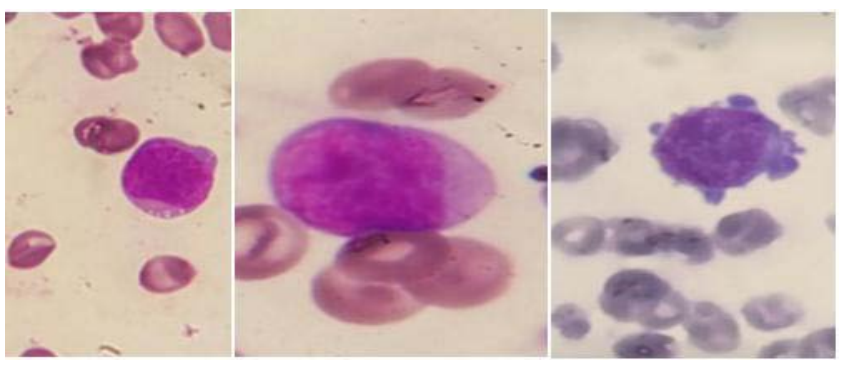

Figure 1: Myeoblasts with different differentiation, some have vacuoles \& fine granules while few have cytoplasmic projections.
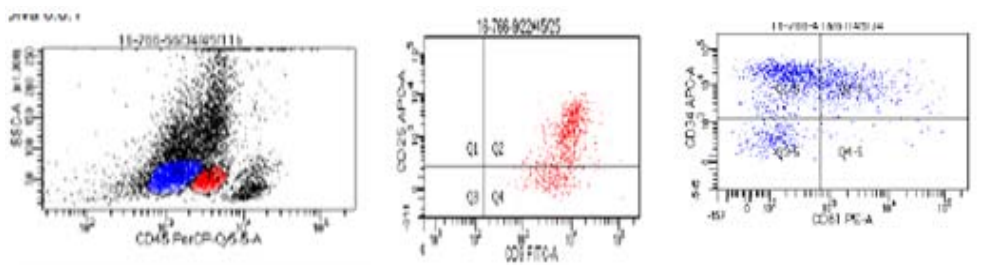

Figure 2: The flowcytometry revealed 2 distinct populations; the red population is the basophilic blasts while the blue one is the myeloblasts with megakaryoblastic differentiation.

of myeloid neoplasms and acute leukemia has introduced new provisional category of AML with positive BCR-ABL1 genes rearrangement [5]. This new category attempted to recognize these rare de novo AML cases that may benefit from TKI therapy [6]. Although the diagnostic distinction between de novo AML with BCR-ABL1 and blast transformation of CML may be difficult without adequate clinical information, the significance of detecting this fusion as a provisional disease category is justified because appropriate targeted therapy may benefit these patients. We actually faced two challenges in this case, first is the finding of the two types of blasts of myeloid lineage. Actually the blasts of basophilic origin couldn't be distinguished easily from regular myeloblasts morphologically since the coarse granules were not seen. Sudan Black was negative and Toulidine Blue stain was not done.

They could be identified by Flowcytometry with their distinct appearance in Basophils gate (i.e. Moderate CD45 which was dimmer than Lymphocytes and brighter than Myeloblast gate) and positivity of the following markers CD34, CD117, CD13, CD33, CD22, and CD9 \& CD25 on this population denoting Basophilic differentiation. Upon reviewing the literature only few rare cases describing such abnormal combination is found.

The second challenge arose when the Fish result showed positive Philadelphia chromosome, the question was whether it is Blast crisis of CML or Denovo AML with positive ph chromosome.

Preliminary data suggest that additional molecular markers such as deletion of antigen receptor genes (IGH, TCR), IKZF1 and/or CDKN2A may support a diagnosis of de novo ABL over blast crisis of CML [7]. Unfortunately those molecular markers are not available in our laboratory. As per the WHO classification 2016, our case could be classified either as AML with BCR-ABL1 positive or CML with Blast crisis with Divergent differentiation of blasts. Our dilemma persists and can only be dispelled with further molecular testing of the above markers.
To sum up, our patient didn't have history of CML or MPN nor splenomegaly. Overall features with presence of $\mathrm{Ph}$ positive chromosome, favored diagnosis of CML in concurrent Megakaryblastic and Basophilic blast crisis over De Novo AML Hybrid (Megakaryblastic/Basophilic) type.

Further, established protocols or guidelines to distinguish these two entities are not readily available in literature. This distinction however is imperative in order to make therapeutic decisions

\section{Conclusion}

The clinical findings, bone marrow examination, FISH and Flowcytometry support the diagnosis of a very rare Acute Myeloid Leukemia with combination of blasts of same Lineage (Megakaryoblast and Basophil Blast).

The definite diagnosis of this case was difficult based exclusively on morphological findings. Flowcytometry, cytogenetic and molecular studies are mandatory in a patient presenting De novo with such findings as the diagnosis cannot be ascertained without them.

Reporting such rare cases is important to be able to understand more regarding their pathophysiology in the future.

Our report is limited by the lack of detailed cytogenetic and molecular analysis, but we aimed to highlight this previously unreported heterogenic morphological combination of Basophilic and Megakaryocytic Differentiation of Blasts in Acute Myeloid Leukemia.

\section{Consent}

Written informed consent was obtained from the patient for publication of this case report \&any accompanying images.

\section{Acknowledgment}

The author of this article acknowledged the Flowcytometry Technologist Tahani Al Nas for her early recognition of abnormal 
immune phenotype.

\section{References}

1. Keung YK, Beaty M, Powell BL, Molnar I, Buss D, Pettenati M. Philadelphia chromosome positive myelodysplastic syndrome and acute myeloid leukemia: retrospective study and review of literature. Leuk Res. 2004; 28: 579-586.

2. Paietta E, Racevskis J, Bennett JM, Neuberg D, Cassileth PA, Rowe JM Biologic heterogeneity in Philadelphia chromosome-positive acute leukemia with myeloid morphology: the Eastern Cooperative Oncology Group experience. Leukemia. 1998; 12: 1881-1885.

3. Gill RM, Etzell JE. Basophilic leukemia in recurrent chronic myelogenous leukemia blast phase. Am J Hematol. 2007; 82: 736-737.

4. Sreejesh sreedharanumni, Narender kumar, Gaurav prakash. Basophilia and megakaryoblastic differentiation in a case of acute myeloid leukemia:
An unusual morphological combination Hematology/Oncology and Stem Cell Therapy. 2016; 9: 30-33.

5. Arber DA, Orazi A, Hasserjian R, Thiele J, Borowitz MJ, Le Beau MM, et al Review Series- The 2016 revision to the World Health Organization (WHO) Classification of myeloid neoplasms and acute leukemia. Blood. 2016; 127: 2391-2405.

6. Soupir CP, Vergilio JA, Dal Cin P, Muzikansky A, Kantarjian H, Jones D. Philadelphia chromosome-positive acute myeloid leukemia: a rare aggressive leukemia with clinicopathologic features distinct from chronic myeloid leukemia in myeloid blast crisis. Am J Clin Pathol. 2007; 127: 642-650.

7. Nacheva EP, Grace CD, Brazma D, Gancheva K, Howard-Reeves J, Rai L. Does BCR/ABL1 positive acute myeloid leukaemia exist? $\mathrm{Br} \mathrm{J}$ Haematol. 2013; 161: 541-550. 